\title{
Trap Model for Clogging and Unclogging in Granular Hopper Flows
}

\author{
Alexandre Nicolas ${ }^{*}$ \\ LPTMS, CNRS, Université Paris-Sud, Université Paris-Saclay, 91405 Orsay, France \\ Ángel Garcimartín and Iker Zuriguel \\ Departamento de Física y Matemática Aplicada, Facultad de Ciencias, Universidad de Navarra, 31080 Pamplona, Spain
}

(Received 26 September 2017; published 11 May 2018)

\begin{abstract}
Granular flows through narrow outlets may be interrupted by the formation of arches or vaults that clog the exit. These clogs may be destroyed by vibrations. A feature which remains elusive is the broad distribution $p(\tau)$ of clog lifetimes $\tau$ measured under constant vibrations. Here, we propose a simple model for arch breaking, in which the vibrations are formally equivalent to thermal fluctuations in a Langevin equation; the rupture of an arch corresponds to the escape from an energy trap. We infer the distribution of trap depths from experiments made in two-dimensional hoppers. Using this distribution, we show that the model captures the empirically observed heavy tails in $p(\tau)$. These heavy tails flatten at large $\tau$, consistently with experimental observations under weak vibrations. But, here, we find that this flattening is systematic, which casts doubt on the ability of gentle vibrations to restore a finite outflow forever. The trap model also replicates recent results on the effect of increasing gravity on the statistics of clog formation in a static silo. Therefore, the proposed framework points to a common physical underpinning to the processes of clogging and unclogging, despite their different statistics.
\end{abstract}

DOI: 10.1103/PhysRevLett.120.198002

When discrete bodies flow through a constriction, there exists a risk of clogging, due to the spontaneous formation of archlike (in two dimensions) or domelike (in three dimensions) structures obstructing the bottleneck. This phenomenon can arise in an impressive variety of systems [1-5] and similar features have been observed in most of them, from granular packings flowing out of a vibrating silo [6] and colloids flowing through an orifice under a pressure gradient [7], to living beings, such as mice [8], sheep [9], and pedestrians [10]. In particular, while the flow intervals $t_{f}$ between clogs are exponentially distributed, the distribution of lifetimes $\tau$ of (temporary) individual clogs is heavy tailed and can be fitted to a power law, viz., $p(\tau) \sim \tau^{-\alpha}$. When the exponent $\alpha$ is smaller than 2, the average clog lifetime $\langle\tau\rangle$ does not converge; the mean outflow thus vanishes, which in practice means that extremely long clogs will dominate the process. This defines the clogged regime [9]. In contrast, a finite mean outflow is obtained for $\alpha>2$, despite the flow intermittency.

In granular hopper flows, the unclogged regime $\alpha>2$ can be reached by enlarging the outlet or by applying stronger vibrations to the setup, both of which lead to larger values of $\alpha$, hence fewer long-lived clogs [9]. It is still debated whether clogs completely disappear above a critical outlet size in the absence of vibrations, or whether an (infinite) static silo will always clog up, eventually [11-14]. Beyond this conceptual question, differences have been put in the limelight between the static case and the shaken one. In particular, the formation of a clog and its destruction through vibrations follow different statistics, which has suggested that these processes are fundamentally distinct [6,15-17]. Indeed, clogging is described as a Poissonian process characterized by a constant probability of formation of a stable arch. On the other hand, the unclogging probability is not constant over time: The longer an arch has survived, the longer it will probably still live. Accordingly, this phenomenon was ascribed to aging [18], but so far this explanation has not been confirmed by experimental evidence.

In this Letter, we promote a different explanation, centered on the heterogeneous native arch stabilities. We put forward a simple model that rationalizes the heavy tails of the unclogging process in vibrated silos. Remarkably, when applied to static silos, the model is found to reproduce several characteristic features, thus hinting at a common underpinning for clogging and unclogging.

Consider the arch sketched in Fig. 1(a), which is subjected to vertical vibrations characterized by a dimensionless acceleration $\Gamma=\left[\left(A \omega^{2}\right) / g\right]$, where $A$ is the
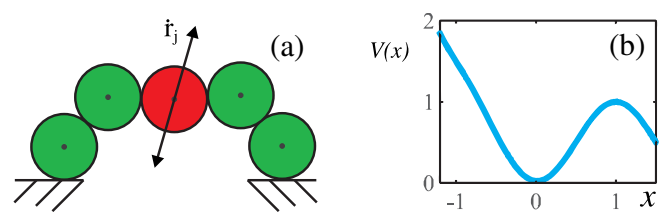

FIG. 1. (a) Sketch of an arch of vibrated grains. (b) Profile of the potential well used in the numerical simulations. 
amplitude and $\omega$ is the angular frequency. Newton's equation of motion for grain $j$ (at position $\boldsymbol{r}_{j}$ ) reads

$$
\ddot{\boldsymbol{r}}_{j}=-\frac{\partial}{\partial \boldsymbol{r}_{j}} V\left(\boldsymbol{r}_{1}, \ldots, \boldsymbol{r}_{N}\right)+\boldsymbol{g}+\boldsymbol{f}_{j}+\xi(t)
$$

where the mass of the grain has been set to one. The first term on the right-hand side accounts for the conservative interactions between grains ( $V$ is the global potential energy), $\boldsymbol{g}$ is the gravity, and $\boldsymbol{f}_{j}$ is a dissipative frictional force. The vibrations induce an extra force $\xi(t)$. Let us focus on the weakest link $j$ in the arch and overlook the deformation of the rest of the arch, whence we approximate $V\left(\boldsymbol{r}_{1}, \ldots, \boldsymbol{r}_{N}\right) \approx V\left(\boldsymbol{r}_{j}\right)$. This is supported by experimental observations indicating that the particle with the largest angle dominates the breaking process [19]. To simplify the picture further, the gravitational potential energy is included in $V$ and we assume quasi-one-dimensional motion $(\boldsymbol{r} \rightarrow x)$, viz., $\ddot{x}=-V^{\prime}(x)+f+\xi(t)$ where the $j$ subscripts have been dropped. As vibrations are symmetric, $\langle\xi(t)\rangle=0$. During the clogging event, $\langle\ddot{x}\rangle \approx 0$ and $V^{\prime}(x)$ evolves much more slowly than $\xi(t)$, so taking the variance of the equation of motion over a small time window yields $\left\langle\ddot{x}^{2}\right\rangle \approx\left\langle\xi^{2}\right\rangle$. Here, we have also assumed that $f$ increases at most linearly with the velocity $|\dot{x}|$ of the grain, so that at high $\omega,\left\langle\ddot{x}^{2}\right\rangle \sim \omega^{2}\left\langle\dot{x}^{2}\right\rangle \gg\left\langle f^{2}\right\rangle$. Finally, we note that the acceleration of the grain must be roughly proportional to the acceleration measured on the vibrated setup, viz., $\left\langle\ddot{x}^{2}\right\rangle=\operatorname{Tr}(\omega, \rho, \ldots) \Gamma^{2}$, where the transmission factor $\operatorname{Tr}$ includes the dependence on the material parameters of the grain, the frequency $\omega$, the density $\rho$, and so on. To leading order, overlooking these dependencies and the possible temporal correlations of the vibrations $\xi(t)$, we arrive at

$$
\ddot{x}=-V^{\prime}(x)+f+\xi(t)
$$

with $\langle\xi(t)\rangle=0$ and $\left\langle\xi(t) \xi\left(t^{\prime}\right)\right\rangle \propto \Gamma^{2} \delta\left(t-t^{\prime}\right)$. We notice that, in the case of viscous friction, i.e., $f \equiv-\gamma \dot{x}$ (with $\gamma$ the drag coefficient), Eq. (1) is a Langevin equation with a vibration-induced Gaussian white noise associated with a temperature $\beta^{-1}=\Gamma^{2} / \gamma$. In the present work, we focus on this analytically tractable case, leaving for a separate study its generalization to other models of friction. The stability of the arch implies that $x$ sits in a basin of $V$, constrained by an energy barrier of height, say, $E_{b}$. The hopping rate over such a barrier was worked out by Kramers [20] and reads

$$
k \equiv\langle\tau\rangle^{-1}=\nu e^{-\beta E_{b}},
$$

where the attempt frequency $\nu$ depends on $\gamma$ and on the angular vibrational frequencies $\omega_{0}$ and $\omega_{b}$ at the minimum and at the saddle point. Kramers's formula holds in the moderate to high damping regime $\gamma>\nu$, for $\beta E_{b} \ll 1$ (hence, $k<\nu$ ). For a single $E_{b}$, hence a single $k$, different realizations of the noise yield an exponential distribution of escape times $\tau$ [21]

$$
p(\tau \mid k)=k e^{-k \tau} .
$$

In reality, energy barriers are expected to take a whole range of values, reflected by a distribution $p\left(E_{b}\right)$. So will then the hopping rates $k$, by virtue of $p(k) d k=p\left(E_{b}\right) d E_{b}$. In this situation, the distribution of escape times $\tau\left(\tau \geq \nu^{-1}\right)$ is given by the convolution

$$
p(\tau)=\int_{0}^{\nu} d k p(\tau \mid k) p(k) .
$$

The remaining step is to gather information on the features of the energy landscape, and more specifically $p\left(E_{b}\right)$. To do so, we exploit the arch-destabilization experiments performed by Lozano et al. [19], in which an acceleration $\operatorname{ramp} \Gamma(t)=\dot{\Gamma} t$ was applied to the arch until it breaks. An arch will typically break at an intensity $\Gamma_{c}$ such that the breaking time $\langle\tau\rangle$ is of the order of the experimental ramp time $\left(\alpha \dot{\Gamma}^{-1}\right)$. Assimilating $\tau$ to the escape time from a trap of depth $E_{b}$ and using Eq. (2) with $\beta=\left(\gamma / \Gamma^{2}\right)$, we get $E_{b} \approx \ln (\nu / \dot{\Gamma}) \Gamma_{c}^{2} / \gamma$. Neglecting the weak (logarithmic) dependences on $\nu$ and $\dot{\Gamma}$, we arrive at

$$
E_{b} \approx \frac{\Gamma_{c}^{2}}{\gamma} .
$$

More rigorous arguments [22] lead to the same scaling. Besides, it was observed that the average value $\Gamma_{c}$ at which arches broke was virtually insensitive to $\dot{\Gamma}$ in a given range [19]. Equation (5) implies that the exponential distributions $p\left(\Gamma_{c}\right)$ measured experimentally for all tested outlet sizes $D$ [23] translate into a Weibull distribution of energy barriers $p\left(E_{b}\right)$, viz.,

$$
E_{b}=E_{b}^{\star} y^{a} \quad \text { with } \quad p(y)=e^{-y}
$$

where $a=2, y \equiv\left(\Gamma_{c} /\left\langle\Gamma_{c}\right\rangle\right)$, and $E_{b}^{\star}$ implicitly depends on $D$.

For more generality, we will nonetheless study Weibull distributions $p\left(E_{b}\right)$ of arbitrary inverse shape parameters $a$. We start the discussion with the simple case $a=1$, i.e., an exponential distribution. Equation (4) then turns into

$$
p(\tau)=\epsilon \int_{0}^{\nu} d k e^{-k \tau}\left(\frac{k}{\nu}\right)^{\epsilon},
$$

where the dimensionless temperature $\epsilon \equiv\left[\Gamma^{2} /\left(\gamma E_{b}^{\star}\right)\right]$ has been introduced.

Changing variables to $\tilde{k} \equiv k \tau$ and rescaling time as $\tau \rightarrow \tilde{\tau} \equiv \nu \tau$, one easily arrives at the probability density function (pdf) for $\tilde{\tau}$,

$$
p(\tilde{\tau})=\frac{\epsilon}{\tilde{\tau}^{1+\epsilon}} \int_{0}^{\tilde{\tau}} d \tilde{k} e^{-\tilde{k}} \tilde{k}^{\epsilon} .
$$

The complementary cumulative distribution function (CCDF) $P(T>\tilde{\tau})$, also called survival function, then reads 

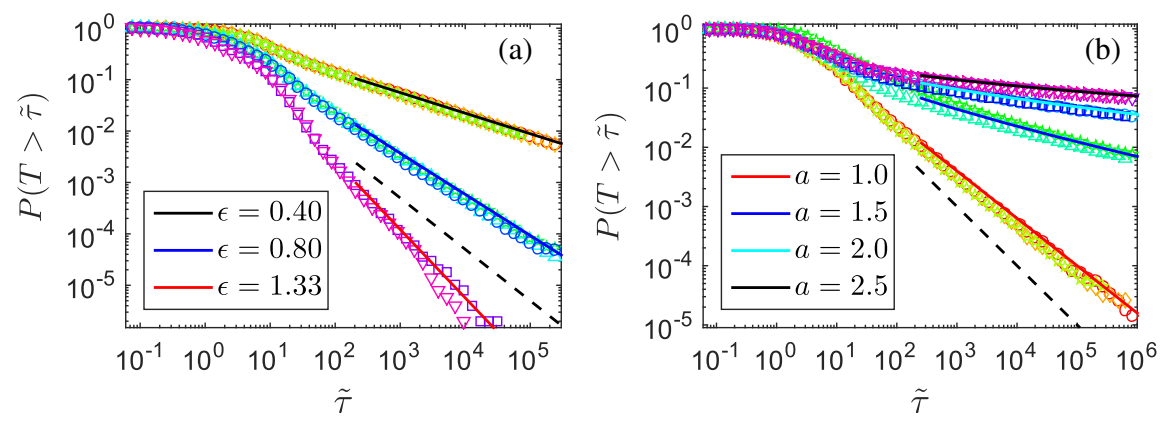

FIG. 2. (a) Survival functions $P(T>\tilde{\tau})$ for an exponential distribution of barrier heights $(a=1)$ as a function of rescaled time $\tilde{\tau}$, for the values of the vibrational temperature $\epsilon$ indicated in the legend. (b) Survival functions $P(T>\tilde{\tau})$ at fixed $\epsilon=0.8$, for different shape parameters $a$ versus $\tilde{\tau}$. In both plots, the different symbols refer to various parameter sets $\left(\gamma, \Gamma, E_{b}^{\star}\right)$. The data collapse onto master curves coinciding with the predictions of Eqs. (7) and (8), shown as thick lines. The dashed lines in black have slope -1 .

$$
\begin{aligned}
\int_{\tilde{\tau}}^{\infty} p(T) d T & =\epsilon \int_{0}^{\infty} d k e^{-k} k^{\epsilon} \int_{\max (k, \tilde{\tau})}^{\infty} d T T^{-1-\epsilon} \\
& =\left(\int_{0}^{\tilde{\tau}} d k e^{-k} k^{\epsilon}\right) \tilde{\tau}^{-\epsilon}+e^{-\tilde{\tau}} \\
& \approx \Gamma(1+\epsilon) \tilde{\tau}^{-\epsilon}
\end{aligned}
$$

where we have introduced the Gamma function $\Gamma$, so

$$
p(\tilde{\tau}) \approx \epsilon \Gamma(1+\epsilon) \tilde{\tau}^{-1-\epsilon} \quad \text { for } \tilde{\tau} \rightarrow \infty .
$$

The distribution of arch-breaking times thus follows a power law with exponent $\alpha=1+\epsilon$ for $\tilde{\tau} \rightarrow \infty$. Therefore, the unclogging transition will be reached by simply increasing $\epsilon$ (it takes place at $\epsilon_{c}=1$ ). Interestingly, the same power-law tail distribution is obtained if a single escape time $\tau$ is assigned to each energy barrier (hence, to each $k$ ), instead of the distribution $p(\tau \mid k)$ of Eq. (3). In this case, the description boils down to Bouchaud's trap model for aging in glasses [24], in which the system hops between exponentially distributed energy traps. Related models were also devised to explain, e.g., the power-law blinking of semiconductor nanocrystals [25].

We test this result against numerical simulations relying on the velocity Verlet algorithm for stochastic dynamics [26]. To this end, a particular potential well has to be specified; we have chosen $V(x)=\left(E_{b} / 2\right)\left[1-\cos (\pi x)+e^{-\pi(1+x)}\right]$ [see Fig. 1(b)] [27]. With this specific choice, the attempt frequency $\nu$ is dependent on $E_{b}$ (via $\omega_{0}$ and $\omega_{b}$ ). To account for this dependence, we invert Eq. (2) to get $E_{b}(\tau) \approx$ $E_{b}^{\star} \epsilon \ln (\nu \tau)$, where $\nu$ is the attempt frequency for $E_{b}=E_{b}^{\star}$, and we substitute $\nu\left[E_{b}(\tau)\right]$ for $\nu$ in the rescaled time $\tilde{\tau}=\nu \tau$. The validity of this approach is endorsed by the coincidence between the prediction of Eq. (7) and the simulation results [Fig. 2(a)].

A similar reasoning for the general case $a>0$ leads to an integral for the CCDF that cannot readily be expressed in closed form. Still, we can resort to the approximation indicated above, i.e., neglecting fluctuations for a given trap depth $E_{b}$ and replacing $p(\tau \mid k)$ with $\delta\left(\tau-k^{-1}\right)$. The approximate CCDF can then be written as

$$
P(T>\tilde{\tau}) \approx P\left[E_{b}(T)>E_{b}(\tilde{\tau})\right] \approx e^{-(\epsilon \ln \tilde{\tau})^{1 / a}} .
$$

The numerical results obtained with the potential $V(x)$ for different values of $a$ [Fig. 2(b)] confirm the accuracy of this expression for long time lapses and thus support the validity of the approximation.

Let us now focus on the value $a=2$ which, as mentioned before, is the one that we inferred from the vibration ramp tests. The CCDF $P(T>\tau)$ for various vibrational temperatures $\epsilon$ are plotted in Fig. 3(a), as a function of nonrescaled time $\tau$. In the experimentally accessible region $\left[P(T>\tau)>10^{-3}\right]$, delimited by the thick box on the figure, the survival functions are well described by power laws with exponents that hint at a transition between a clogged regime $(\alpha \leq 2$, diverging $\langle\tau\rangle)$ and an unclogged regime $(\alpha>2$, converging $\langle\tau\rangle)$, consistently with experimental findings.

In fact, one may even directly compare these results with experimental data, by assessing the arch stability $\left\langle\Gamma_{c}\right\rangle$ entering the vibrational temperature $\epsilon \sim \Gamma^{2} /\left\langle\Gamma_{c}\right\rangle^{2}$ thanks to the ramp tests described previously. Figure 4 shows an example of such a comparison for a relatively narrow outlet; another example is provided as Supplemental Material [28], along with further details. Given the simplicity of the model, the agreement, albeit imperfect, is deemed satisfactory. In particular, the slope of the survival function gets steeper (larger $\alpha$ ) when $\epsilon$ increases, as a result of either a higher vibration intensity $\Gamma$ or a larger outlet, hence, a smaller $\left\langle\Gamma_{c}\right\rangle$. The model also captures the flattening of $P(T>\tau)$ at large $\tau$, observed experimentally for narrow apertures or weak vibrations (especially at high frequencies) [23], i.e., at small $\epsilon$. But, remarkably, the model suggests that this flattening is a generic property of gently shaken flows, which arises because of the heavier than exponential tail in $p\left(E_{b}\right)$. 


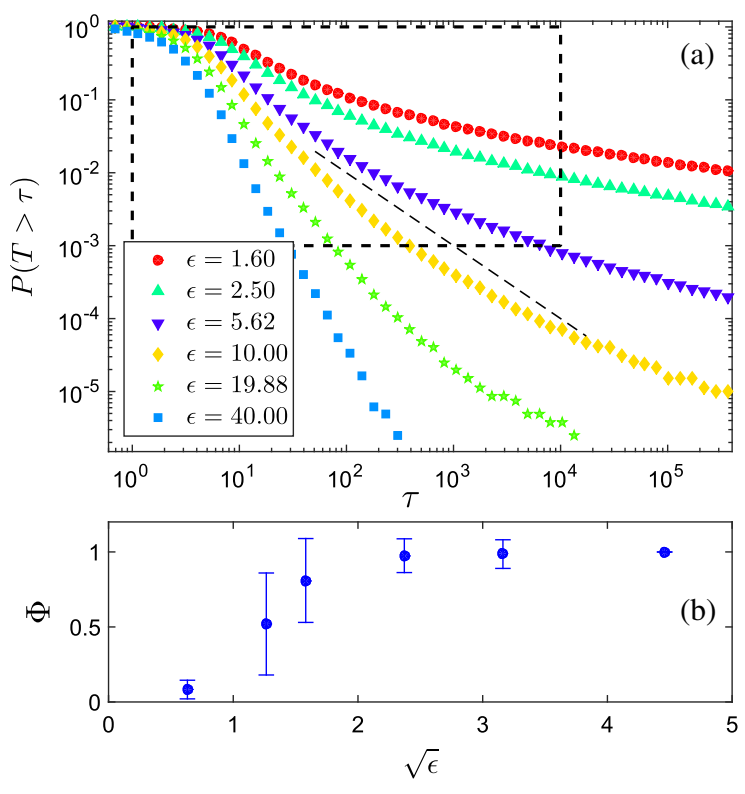

FIG. 3. (a) Survival functions $P(T>\tau)$ as a function of dimensional time $\tau$ for $a=2, \gamma=0.1, E_{b}^{\star}=1$, and different $\epsilon$ as indicated in the legend. The box in thick dashed line indicates the experimentally accessible values. The thin dashed line has slope -1 . (b) Duty cycle $\Phi$ (see main text) calculated over a time window of $1000 \mathrm{~s}$ as a function of the vibrational acceleration $\sqrt{\epsilon} \sim \Gamma$, using the same parameters as in (a). For this calculation, the flow intervals were set to $t_{f}=10 \mathrm{~s}$ and the model time unit was set to $1 \mathrm{~ms}$, to get closer to the experimental values of [6]. The error bars represent standard deviations.

The flattening of the survival function has crucial implications for the unclogging transition exposed above. Indeed, we notice from Eq. (8) that $\langle\tau\rangle=\int_{0}^{\infty} P(T>\tau) d \tau$ diverges for any $a>1$, regardless of the vibrational temperature $\epsilon$. Therefore, the model predicts that the system is always in the clogged regime, provided that the aperture gives rise to exponentially distributed arch stabilities $\Gamma_{c}$ [Eq. (6) with $a=2$ ]. The reasoning holds as long as (i) there is no upper cutoff in $p\left(E_{b}\right)$ in the range of experimentally relevant values and (ii) vibrations are weak enough to not affect the granular density near the outlet, thus leaving $p\left(E_{b}\right)$ mostly unaltered as compared to the vibrationless situation [17]. On no account does this conclusion prevent experiments of finite duration $T$ from appearing to be in the flowing state (if the shaking is vigorous enough). Indeed, the duty cycle $\Phi \equiv\left\langle t_{f}\right\rangle /\left(\left\langle t_{f}\right\rangle+\langle\tau\rangle\right)$, which quantifies the fraction of time that the system spends effectively flowing, will reach finite values (intermittent flow) at high enough $\epsilon$, if it is computed within a temporal window of finite duration $T$. The example plotted in Fig. 3(b) for $T=1000 \mathrm{~s}$ is in fact very similar to the measurements by Janda et al. (see Fig. 7 of [6], where $1-\Phi$ is plotted).

Now that several features of the unclogging process in vibrated silos have been recovered, let us extend the model to granular flows in static silos. When the system is
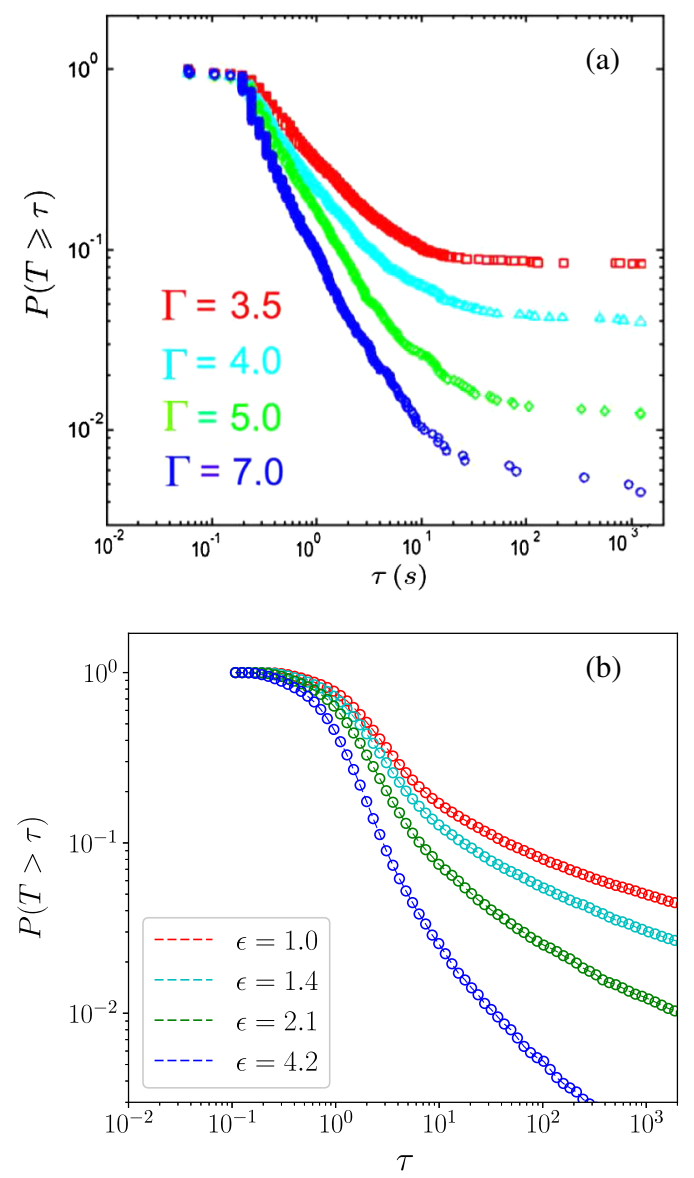

FIG. 4. Comparison between the model and experiments. Survival functions $P(T \geq \tau)$ of clog durations $\tau$ (a) in Lozano's two-dimensional experiments at a vibration frequency of $1 \mathrm{kHz}$ for an aperture of width $D=4.2$ and (b) in our trap model, with $\gamma=0.3$ and $E_{b}^{\star}=1$. (a) was adapted from [29] (p. 101). The model time unit was set to $0.2 \mathrm{~s}$ to facilitate the comparison. Both panels are plotted with identical axes.

flowing, the motion of grains creates an internal agitation, with a kinetic temperature proportional to the kinetic energy per grain: $T_{K} \propto E_{K}$. Using this temperature in our trap model, we expect the system to escape almost immediately from shallow traps $E_{b}^{(j)} \leq E_{K}$, where $j=$ $1 \ldots s-1$ numbers the successive energy barriers. Only when a barrier of height $E_{b}^{(s)}>E_{K}$ is finally encountered will the system be arrested in the trap; without external agitation this halt will last forever. The clogging probability $p_{c}$ per grain is then the probability to encounter such a high barrier, $p_{c}=P\left(E_{b}>E_{K}\right)$. Furthermore, under the assumption that the $E_{b}^{(j)}$ are uncorrelated, the number $s$ of grains that have escaped prior to clogging follows a Bernoulli process. This naturally leads to an exponential distribution of avalanches between clogs, with a mean size $\langle s\rangle=p_{c}^{-1}$ for $p_{c} \ll 1$.

In a recent numerical work, Arevalo et al. computed the avalanche size for different values of gravity $g_{\text {eff }}$ [30]. 
Besides confirming the expected scaling $E_{K} \propto g_{\text {eff }}$ and showing that $E_{K}$ is a prominent parameter for the description of the flow, their main result is the scaling law $\ln \langle s\rangle \propto \sqrt{g_{\text {eff }}}$. From these relations, we arrive at

$$
P\left(E_{b}>E_{K}\right)=\exp \left(-b \sqrt{E_{K}}\right),
$$

where $b$ is a positive constant. Strikingly, the pdf derived from this CCDF takes the form

$$
p\left(E_{b}\right) \sim \sqrt{\frac{E_{b}^{\star}}{E_{b}}} \exp \left(\sqrt{\frac{E_{b}}{E_{b}^{\star}}}\right) .
$$

This is a Weibull distribution with exactly the same shape $(a=2)$ as the one we inferred from ramp experiments in a vibrated silo. Thus, the distribution of energy barriers obtained in a vibrated silo is compatible with the avalanche size dependence on gravity in a static one.

In summary, we have put forward a model which likens unclogging at a bottleneck to the exploration of a simple energy landscape. We derived the statistics of escape times for a generic distribution of energy barriers. For the specific distribution inferred from measurements of arch stabilities, the escape time statistics are consistent with the heavy tails characteristic of flows through bottlenecks. An abundance of extremely long-lived clogs emerges generically in the model and it is thus suggested that gentle vibrations may not restore a permanent steady flow. The model is then extended to static silos, in which clogs persist forever if they can resist until the kinetic energy of all the grains is fully dissipated. We find that the variations of avalanche sizes with gravity reported recently stem from the very same distribution of barriers as that obtained from experiments in vibrated silos. This relation challenges the widespread idea that clogging and unclogging are independent processes that require separate interpretations [31].

A. N. is funded by the Centre National de la Recherche Scientifique. A. G. and I. Z. acknowledge funding from Ministerio de Economía y Competitividad (Spanish Government) through Projects No. FIS2014-57325 and No. FIS2017-84631, MINECO/AEI/FEDER, UE. We would like to thank Ignacio Pagonabarraga, Marie Chupeau, and Ricardo Brito for discussions.

*alexandre.nicolas@polytechnique.edu

[1] D. Helbing, I. Farkas, and T. Vicsek, Nature (London) 407, 487 (2000).

[2] K. To, P.-Y. Lai, and H. K. Pak, Phys. Rev. Lett. 86, 71 (2001).
[3] M. Delarue, J. Hartung, C. Schreck, P. Gniewek, L. Hu, S. Herminghaus, and O. Hallatschek, Nat. Phys. 12, 762 (2016).

[4] M. Haw, Phys. Rev. Lett. 92, 185506 (2004).

[5] D. Genovese and J. Sprakel, Soft Matter 7, 3889 (2011).

[6] A. Janda, D. Maza, A. Garcimartín, E. Kolb, J. Lanuza, and E. Clément, Europhys. Lett. 87, 24002 (2009).

[7] R. C. Hidalgo, A. Goñi-Arana, A. Hernández-Puerta, and I. Pagonabarraga, Phys. Rev. E 97, 012611 (2018).

[8] P. Lin, J. Ma, T. Liu, T. Ran, Y. Si, and T. Li, Physica (Amsterdam) 452A, 157 (2016).

[9] I. Zuriguel, D. R. Parisi, R. C. Hidalgo, C. Lozano, A. Janda, P. A. Gago, J. P. Peralta, L. M. Ferrer, L. A. Pugnaloni, E. Clément et al., Sci. Rep. 4, 7324 (2014).

[10] J. M. Pastor, A. Garcimartín, P. A. Gago, J. P. Peralta, C. Martín-Gómez, L. M. Ferrer, D. Maza, D. R. Parisi, L. A. Pugnaloni, and I. Zuriguel, Phys. Rev. E 92, 062817 (2015).

[11] I. Zuriguel, A. Garcimartín, D. Maza, L. A. Pugnaloni, and J. M. Pastor, Phys. Rev. E 71, 051303 (2005).

[12] K. To, Phys. Rev. E 71, 060301 (2005).

[13] C. C. Thomas and D. J. Durian, Phys. Rev. E 87, 052201 (2013).

[14] C. C. Thomas and D. J. Durian, Phys. Rev. Lett. 114, 178001 (2015).

[15] K. To and H.-T. Tai, Phys. Rev. E 96, 032906 (2017).

[16] I. Zuriguel, Á. Janda, R. Arévalo, D. Maza, and Á. Garcimartín, in EPJ Web of Conferences, Vol. 140 (EDP Sciences, Les Ulis, 2017), p. 01002.

[17] C. Mankoc, A. Garcimartín, I. Zuriguel, D. Maza, and L. A. Pugnaloni, Phys. Rev. E 80, 011309 (2009).

[18] B. Blanc, J.-C. Géminard, and L. Pugnaloni, Eur. Phys. J. E 37, 112 (2014).

[19] C. Lozano, G. Lumay, I. Zuriguel, R. C. Hidalgo, and A. Garcimartín, Phys. Rev. Lett. 109, 068001 (2012).

[20] H. Kramers, Physica (Amsterdam) 7, 284 (1940).

[21] P. Hänggi, P. Talkner, and M. Borkovec, Rev. Mod. Phys. 62, 251 (1990).

[22] See Supplemental Material at http://link.aps.org/ supplemental/10.1103/PhysRevLett.120.198002 for more rigorous arguments.

[23] C. Lozano, I. Zuriguel, and A. Garcimartín, Phys. Rev. E 91, 062203 (2015).

[24] J.-P. Bouchaud, J. Phys. I (France) 2, 1705 (1992).

[25] R. Verberk, A. M. van Oijen, and M. Orrit, Phys. Rev. B 66, 233202 (2002).

[26] N. Grønbech-Jensen and O. Farago, Mol. Phys. 111, 983 (2013).

[27] Another potential function, $V(x)=\left(E_{b} / 8\right)\left\{[1-\cos (\pi x)]^{3}+\right.$ $\left.4 e^{-\pi(1+x)}\right\}$, was tested and was found to yield almost identical results.

[28] See Supplemental Material at http://link.aps.org/ supplemental/10.1103/PhysRevLett.120.198002.

[29] C. Lozano, Ph.D. thesis, Universidad de Navarra, 2014.

[30] R. Arévalo and I. Zuriguel, Soft Matter 12, 123 (2016).

[31] Also see C. Merrigan, S. K. Birwa, S. Tewari, and B. Chakraborty, Phys. Rev. E 97, 040901(R) (2018). 